\title{
The Electric Power Grid: Today and Tomorrow
}

\author{
Massoud Amin (University of Minnesota, USA) \\ John Stringer (Electric Power Research Institute, USA, retired)
}

\begin{abstract}
In the coming decades, electricity's share of total global energy is expected to continue to grow, and more intelligent processes will be introduced into the electric power delivery (transmission and distribution) networks. It is envisioned that the electric power grid will move from an electromechanically controlled system to an electronically controlled network in the next two decades. A key challenge is how to redesign, retrofit, and upgrade the existing electromechanically controlled system into a smart self-healing grid that is driven by a well-designed market approach. Revolutionary developments in both information technology and materials science and engineering promise significant improvements in the security, reliability, efficiency, and cost effectiveness of electric power delivery systems. Focus areas in materials and devices include sensors, smart materials and structures, microfabrication, nanotechnology, advanced materials, and smart devices.
\end{abstract}

\section{Introduction}

Electric power systems constitute the fundamental infrastructure of modern society. Often continental in scale, electric power grids and distribution networks reach virtually every home, office, factory, and institution in developed countries and have made remarkable, if remarkably insufficient, penetration in developing countries or emerging economies such as China and India (see Figure 1).

The electric power grid can be defined as the entire apparatus of wires and machines that connects the sources of electricity (i.e., the power plants) with customers and their myriad needs. ${ }^{1-5}$ Power plants convert a primary form of energy, such as the chemical energy stored in coal, the radiant energy in sun-

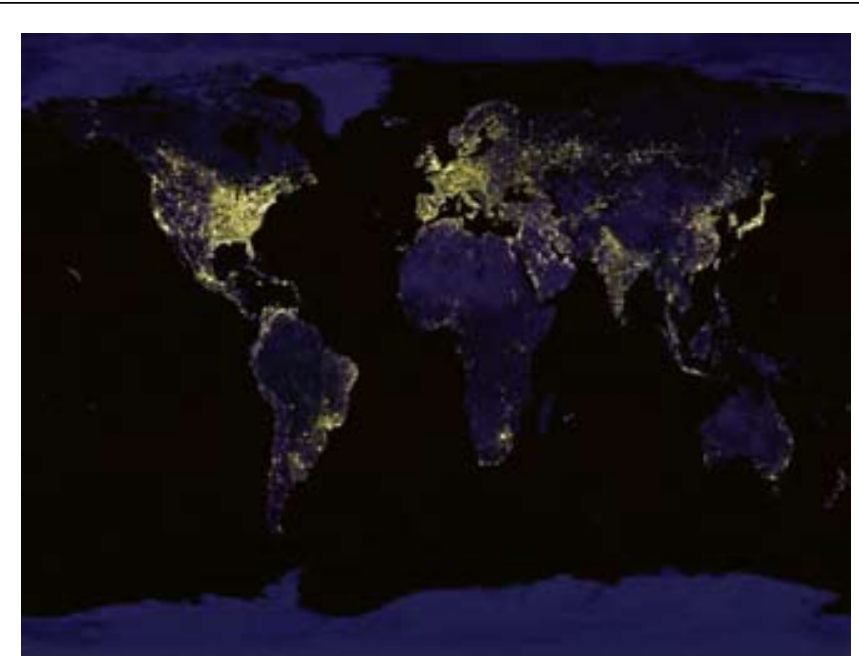

Figure 1. Power grids across the world as seen by nighttime satellite photograph of artificial lighting. Source: U.S. National Aeronautics and Space Administration (NASA); http://antwrp.gsfc.nasa.gov/apod/ ap001127.html (accessed January 2008). light, the pressure of wind, or the energy stored at the core of uranium atoms, into electricity, which is no more than a temporary, flexible, and portable form of energy. It is important to remember that electricity is not a fuel: it is an energy carrier. At the end of the grid, at factories and homes, electricity is transformed back into useful forms of energy or activity, such as heat, light, information processing, or torque for motors.

From a historical perspective, the electric power system in the United States evolved in the first half of the 20th century without a clear awareness and analysis of the system-wide implications of its evolution. In 1940, 10\% of the energy consumption in the United States was used to produce electricity. By 1970 , this had risen to $25 \%$, and by 2002 , it had risen to $40 \%$. (Worldwide, current electricity production is near 15,000 billion kilowatt-hours $(\mathrm{kWh})$ per year, with the United States, Canada, and Mexico responsible for about $30 \%$ of this consumption.) This grid now underlies every aspect of our economy and society, and it has been hailed by the National Academy of Engineering as the 20th century's engineering innovation most beneficial to our civilization. The role of electric power has grown steadily in both scope and importance during this time, and electricity is increasingly recognized as a key to societal progress throughout the world, driving economic prosperity and security and improving the quality of life. Still, it is noteworthy that, at the time of this writing, about 1.4 billion people in the world have no access to electricity, and another 1.2 billion people have inadequate access to electricity (meaning that they experience outages of $4 \mathrm{~h}$ or longer per day).

Once "loosely" interconnected networks of largely local systems, electric power grids increasingly host large-scale, longdistance wheeling (movement of wholesale power) from one region or company to another. Likewise, the connection of distributed resources, primarily small generators, is growing rapidly. The extent of interconnectedness, like the number of sources, controls, and loads, has grown with time. In terms of the sheer number of nodes, as well as the variety of sources, controls, and loads, electric power grids are among the most complex networks made. 
In the coming decades, electricity's share of total energy is expected to continue to grow, as more efficient and intelligent processes are introduced into this network. Electric power is expected to be the fastest-growing source of end-use energy supply throughout the world. To meet global power projections, it is estimated by the Energy Information Administration (EIA) of the U.S. Department of Energy (DOE) that over \$1 trillion will have to be spent during the next 10 years. The electric power industry has undergone a substantial degree of privatization in a number of countries over the past few years..$^{6-9}$ Growth in power generation is expected to be particularly strong in the rapidly growing economies of Asia, with China leading the way (see Figure 2).

In addition, the electricity grid faces (at least) three looming challenges: its organization, its technical ability to meet 25 - and 50 -year electricity needs, and its capacity to increase efficiency without diminishing reliability and security. ${ }^{1-5,8-14}$

The technical aspects of the challenges that will be posed by this rapid growth include both improving existing technology through engineering and inventing new technologies requiring new materials. Some materials advances will improve present technology (e.g., stronger, higher current overhead lines), some will enable emerging technology (e.g., superconducting cables, fault current limiters, and transformers), and some will anticipate technologies that are still conceptual (e.g., storage for extensive solar or wind energy generation).

\section{The Grid}

When most people talk about the "grid," they are usually referring to the electrical transmission system, which moves the electricity from power plants to substations located close to large groups of users. ${ }^{28-29}$ However, the grid also encompasses the distribution facilities that move the electricity from the substations to the individual users.

In the United States alone, the electrical network includes some 15,000 generators with an average thermal efficiency of approximately $33 \%$ at 10,000 power plants. These generators send power through 211,000 miles $(339,000 \mathrm{~km})$ of highvoltage (HV) transmission lines. In addition, there are about 5,600 distribution facilities. In 2002, the installed generating capacity in the United States was $981,000 \mathrm{MW}$. If the power plants ran full time, the net annual generation would be 8,590 $\times 10^{6} \mathrm{kWh}$; the actual net generation was $3,840 \times 10^{6} \mathrm{kWh}$, representing a "capacity factor" of $44.7 \%$.

Currently, North America operates about 211,000 miles $(339,000 \mathrm{~km})$ of high voltage $(>230 \mathrm{kV})$ electric transmission lines. Whereas electricity demand increased by about $25 \%$ since 1990, construction of transmission facilities decreased by about $30 \%$, based on information from the DOE Office of Electricity Delivery and Energy Reliability (OE). The planned transmission lines ( $230 \mathrm{kV}$ or greater) for the period 2004-2013 total approximately 7,000 miles $(11,000 \mathrm{~km}) .{ }^{15}$ According to the
EIA, $281 \mathrm{GW}$ of new generating capacity will be needed by 2025 to meet the growing demand for electricity; on the basis of current needs, this implies a need for about 50,000 miles $(80,000 \mathrm{~km})$ of new HV transmission lines.

As currently configured, the continental-scale grid is a multiscale, multilevel hybrid system consisting of vertically integrated hierarchical networks including the generation layer and the following three basic levels: ${ }^{16-25}$

- transmission level, consisting of meshed networks combining extra high voltage (above $300 \mathrm{kV}$ ) and high voltage $(100-300 \mathrm{kV})$, connected to large generation units and very large customers and, via tie lines, to neighboring transmission networks and to the subtransmission level;

- subtransmission level, consisting of a radial or weakly coupled network including some high voltage (100-300kV) but typically medium voltage $(5-15 \mathrm{kV})$, connected to large customers and medium-size generators; and

- distribution level, typically consisting of a tree network including low voltage (110-115 V or 220-240 V) and medium voltage $(1-100 \mathrm{kV})$, connected to small generators, medium-size customers, and local low-voltage networks for small customers.

In its adaptation to disturbances, a power system can be characterized as having multiple states, or "modes," during which specific operational and control actions and reactions are taking place. ${ }^{17-19}$ These modes can be described as normal, involving economic dispatch, load frequency control, maintenance, and forecasting, for example; disturbance, involving, for instance, faults, instability, and load shedding; and restorative, involving rescheduling, resynchronization, and load restoration, for example.

In addition to these spatial, energy, and operational levels, power systems are also multiscale in the time domain, from nanoseconds to decades, as shown in Table I.

Why the need for a system of such daunting complexity? In principle, it might seem possible to satisfy a small user groupfor example, a small city - with one or two generator plants. However, the electricity supply system has a general objective 
Table I: Multiscale Time Hierarchy of Power Systems.

\section{Action/Operation}

Wave effects (fast dynamics, lightning-caused overvoltages)

Switching overvoltages
Fault protection
$\begin{aligned} & \text { Electromagnetic effects in } \\ & \text { machine windings }\end{aligned}$
Stability

Stability augmentation

Electromechanical effects of generators

Tie line load frequency control Economic load dispatch

Thermodynamic changes from boiler control action (slow dynamics)

System structure monitoring (what is energized and what is not)

System state measurement and estimation

System security monitoring

Load management, load

forecasting, generation scheduling

Maintenance scheduling

Expansion planning

Power plant site selection, design, construction, environmental impact, etc. oscillations in motors and

\section{Time Frame}

Microseconds to milliseconds

Milliseconds

$100 \mathrm{~ms}$ or a few cycles

Milliseconds to seconds

60 cycles or 1 second

Seconds

Milliseconds to minutes

1-10 s; ongoing

10 s to 1 hour; ongoing

Seconds to hours

Steady state; ongoing

Steady state; ongoing

Steady state; ongoing

$1 \mathrm{~h}$ to 1 day or longer; ongoing

Months to 1 year; ongoing

Years; ongoing

2-10 years or longer

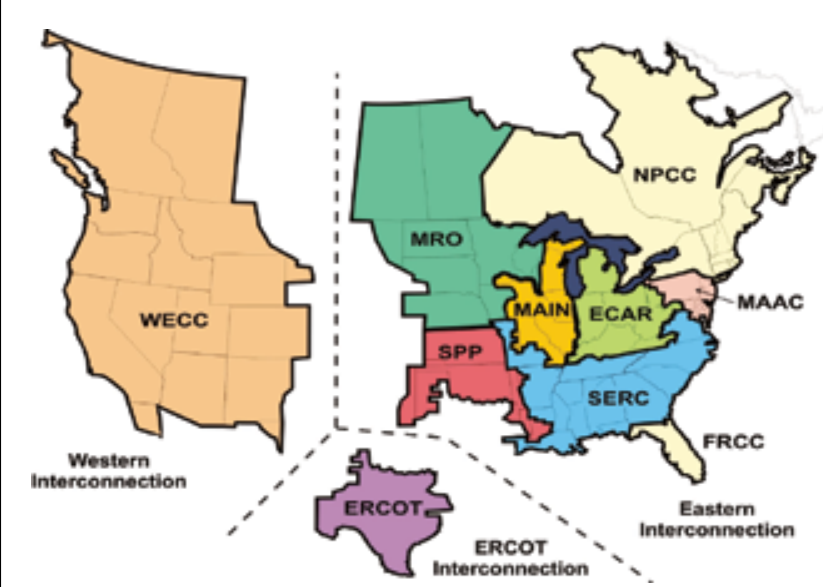

Figure 3. The three grid interconnections in North America. Note: ECAR, East Central Area Reliability Coordination Agreement; ERCOT, Electric Reliability Council of Texas; FRCC, Florida Reliability Coordination Council; MAAC, Mid-Atlantic Area Council; MAIN, MidAmerica Interpool Network; MRO, Midwest Reliability Organization; NPCC, Northeast Power Coordinating Council; SERC, Southeastern Electric Reliability Council; SPP, Southwest Power Pool; WECC, Western Electricity Coordinating Council. of very high reliability, and that is not possible with a small number of generators. This is what led the industry to the existing system in North America where there are just three "interconnects" (Figure 3). Within each of these interconnects, all generators are tightly synchronized, and any failure in a generator immediately is covered from other parts of the system. The interconnects are the Eastern, covering the eastern twothirds of the United States and Canada; the Western, covering the rest of the two countries except Texas; and the Electric Reliability Council of Texas (ERCOT) covering most of Texas. The interconnects have limited direct-current (dc) links between them.

One of the important issues with the use of electricity is that the storage of electricity is very difficult, so the generation and use must be matched continuously. This means that generators must be dispatched as needed, and the United States power grids have approximately 150 control area operators using computerized control centers. Generally, generators are classified as baseload, which are run all the time to supply the minimum demand level; peaking, which are run only to meet power needs at maximum load; and intermediate, which handle the rest. Actually, the dispatch order is much more complicated than this, because of the variation in customer demand from day to night and from season to season.

\section{Electrical Transmission Lines: Challenges and Materials Solutions}

Transmitting electric power over large distances can result in losses up to $7.5 \%$. The major loss is heat, which can be reduced by increasing the voltage and decreasing the current. Most of the power in the United States is alternating current (ac), and this allows the power from the generator to be stepped up. This is done using transformers, in a unit called a step-up transmission substation. Long-distance transmission is typically done through overhead lines with voltages of $110-765 \mathrm{kV}$. The capacity of an overhead line varies with the voltage and the distance: thus, a $765 \mathrm{kV}$ line with a 100 -mile (160-km) length has a maximum capacity of $3.8 \mathrm{GW}$, whereas for a 400 -mile $(640-\mathrm{km})$ length, the capacity is $2.0 \mathrm{GW}$. To avoid system failures, the amount of power flowing over each transmission line must remain below the line's capacity.

The principal limitation on the capacity of a line is its temperature. As a line gets warmer, it sags, and in the worst cases, it can touch trees or the ground. Another factor is the mechanical strength of the support structure. Conductors with higher strength-to-weight ratios for a given current-carrying capacity can increase the overall capacity of the right-of-way. Typically, the right-of-way for a $230 \mathrm{kV}$ transmission line is $75-150$ feet $(23-46 \mathrm{~m})$ or more.

The standard material for overhead conductors in transmission systems is aluminum conductor steel reinforced (ACSR), ${ }^{26}$ which consists of fibers of aluminum twisted around a core of steel fibers. The steel core provides the mechanical strength, and the aluminum provides the electrical conductivity. A number of alternative composite cable materials have been developed over the past several years; ${ }^{27}$ the basic candidate composite materials for the substitution of core support members include 1350 H19 aluminum, stainless steel, S-2 glass fibers, E glass fibers, epoxy, T-300 carbon fibers, and Kevlar 49 fibers. For example, a composite formed of a polyester with $54 \mathrm{vol} \%$ of an E-glass fiber as a unidirectional satin cloth was tested. More recently, $3 \mathrm{M}$ has been developing and Oak Ridge National Laboratory (ORNL) has been testing designs of advanced overhead cables, using a composite core in place of the steel; this is an aluminum metal matrix containing Nextel fibers. The conductor wires are made of an aluminum-zirconium alloy; the zirconium precipitates, 


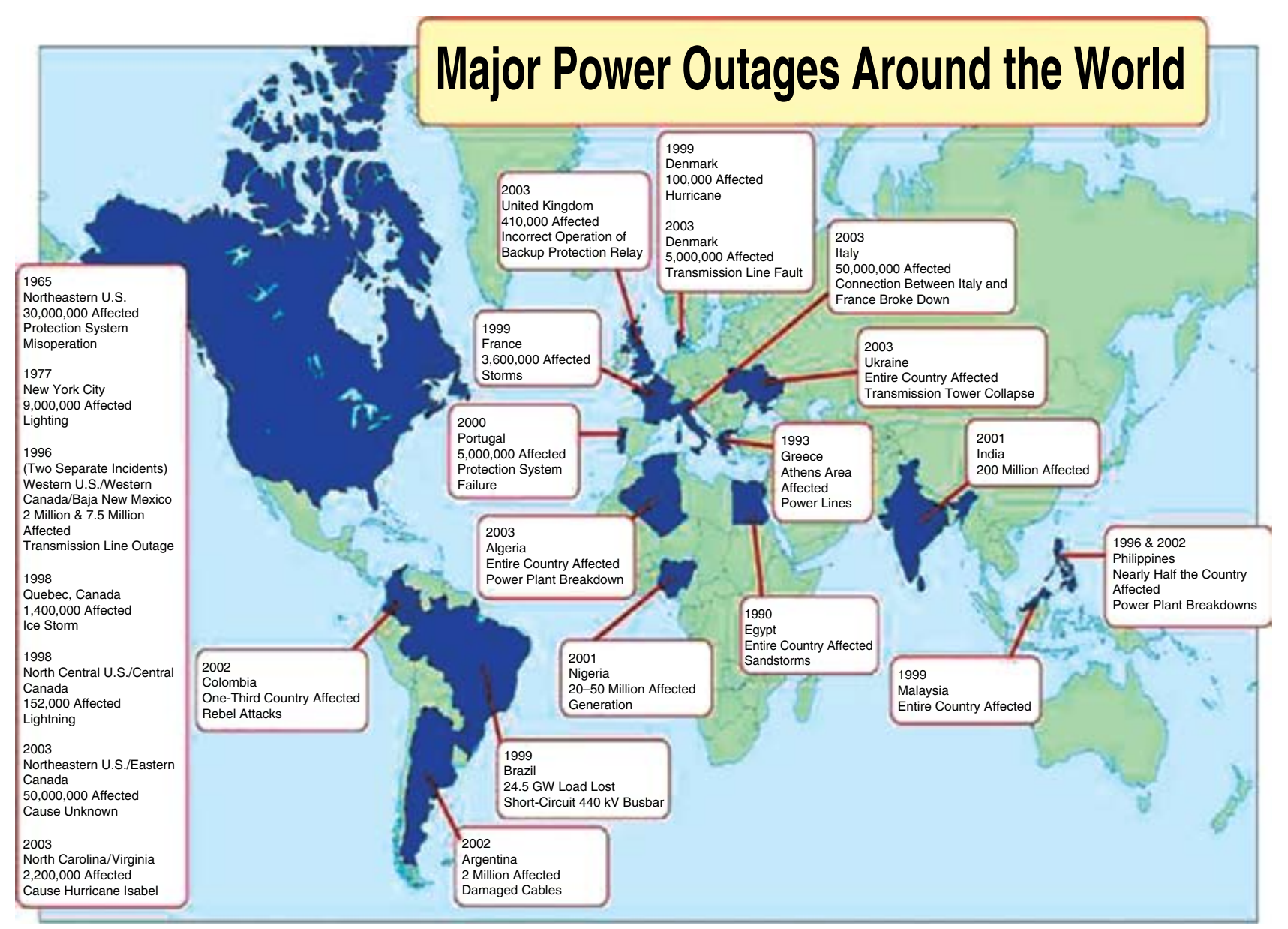

Figure 5. Electrical outages worldwide. Small-scale power outages are not shown. Source: EPRI PEAC Corporation; for more information on blackouts go to www.2003blackout.info (accessed January 2008).

providing a dispersed strengthening to essentially pure (and thus high-conductivity) aluminum. Kirby ${ }^{26}$ points out that the improved composite conductor substituted for the traditional ACSR in an existing transmission line could carry up to three times the current without the need for tower modification or additional rights-of-way. The current objective is to develop a conductor to increase the capacity of existing corridors at five times that of ACSR at current cost by 2010. The ultimate stretch goal is to achieve transmission corridor power densities for cables and conductors of 50 times that of ACSR by 2025 (GridWorks).

\section{Chief Grid Problems}

It is inevitable that an electrical grid built on such a huge scale in a patchwork manner over 100 years will have reliability issues. ${ }^{28,29}$ Several cascading failures during the past 40 years spotlighted the need to understand the complex phenomena associated with power network systems and the development of emergency controls and restoration (Figures 4 and 5). In addition to mechanical failures, overloading a line can create powersupply instabilities such as phase or voltage fluctuations. For an ac power grid to remain stable, the frequency and phase of all power generation units must remain synchronous within narrow limits. A generator that drops $2 \mathrm{~Hz}$ below $60 \mathrm{~Hz}$ will rapidly build up enough heat in its bearings to destroy itself, so circuit

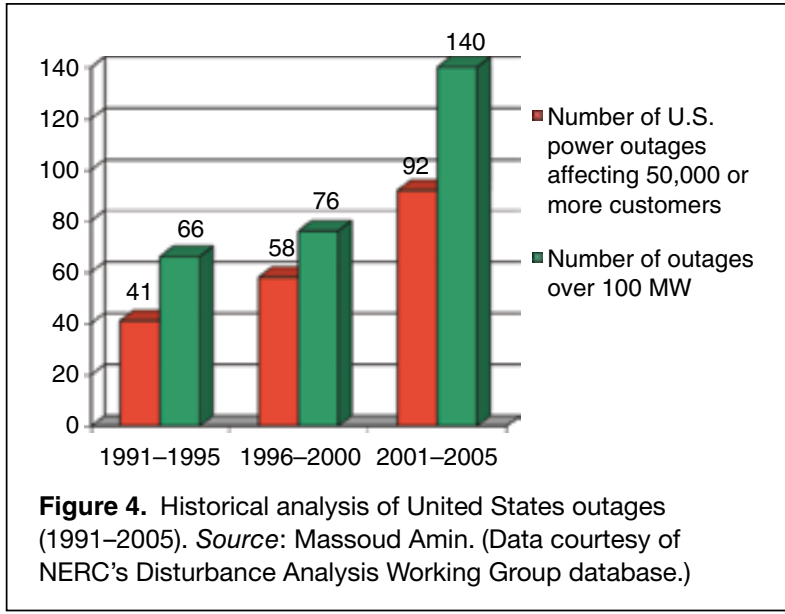

breakers trip a generator out of the system when the frequency varies too much. However, much smaller frequency changes can indicate instability in the grid: in the Eastern Interconnect, a $30 \mathrm{mHz}$ drop in frequency reduces power delivered by $1 \mathrm{GW} .{ }^{30}$

Transmission and distribution losses in the United States were about $5 \%$ in 1970 and grew to $9.5 \%$ in 2001 , as a result of heavier 
utilization and more frequent congestion. ${ }^{31}$ In addition, it is estimated that power outages and power quality disturbances cost the economy from $\$ 75$ to $\$ 180$ billion annually. ${ }^{8,20,32}$ Answers to this challenge require a balanced, risk-managed, and cost-effective approach to investments and use of technology that can make a sizable difference in mitigating the risk. Revolutionary developments in both information technology and materials science and engineering promise significant improvement in the security, reliability, efficiency, and cost effectiveness of all critical infrastructures. Steps taken now can ensure that this critical infrastructure continues to support population growth and economic growth without environmental harm..$^{8,9,12-14}$

Considering their impact, regulatory agencies should be able to induce electricity producers to plan and fund the process. That might be the most efficient way to get it into operation. The current absence of a coordinated national decision-making entity is a major obstacle. States' rights and state public utility commission (PUC) regulations have removed the motivation for individual states' utilities to participate in a national plan. Investor utilities face either collaboration on a national level or a forced nationalization of the industry.

Simply replicating the existing system through expansion or replacement will not only be technically inadequate to meet the changing demands for power, but will also produce a significantly higher price tag for electricity. Through the transformative technologies outlined here, the United States can put in place a 21 st century power system capable of eliminating critical vulnerabilities while meeting intensified consumer demands and, in the process, save society considerable expense.

A phased approach to system implementation will allow utilities to capture many cost synergies. Equipment purchased, for example, should emphasize switchgear, regulators, transformers, controls, and monitoring equipment that can be easily integrated with automated transmission and distribution systems. Long-term plans for equipment upgrades should also address system integration considerations.

Energy policy and technology development and innovation require long-term commitments as well as sustained and patient investments in innovation, technology creation, and development of human capital. Given economic, societal, and quality-of-life issues and the pivotal role of the electricity infrastructure, a selfhealing grid is essential.

\section{Self-Healing Smart Grid}

Controlling a heterogeneous, widely dispersed, yet globally interconnected system is a serious technological challenge. It is even more complex and difficult to control such a system for optimal efficiency and maximum benefit to the consumers while still allowing all its business components to compete fairly and freely. Our proposed strategic vision for achieving this end involves a self-healing "smart grid," an "electrinet," extending to a decade or longer, that would enable more secure and robust systems operation, security monitoring, and efficient energy markets. Such a smart grid would be intelligent, comprising an autonomous digital system capable of identifying surges, downed lines, and outages; resilient, or "self-healing," providing instantaneous damage control; flexible, capable of accommodating new off-grid alternative energy sources; reliable, providing dynamic load balancing; and secure, minimizing vulnerability to terrorist or other attacks. ${ }^{2-4,8,12,20-25,28,29}$

The first step in making the smart self-healing grid is to build a processor into each component of a substation. That is, each breaker, switch, transformer, and busbar, for example, should have an associated processor that can communicate with other such devices. Each high-voltage connection to the device must have a parallel information connection. These processors contain permanent information on device parameters as well as device status and analog measurements from sensors built into the component. Implementing this step will require both existing and new technologies.

When a new device is added to a substation, it will automatically report to the central control computers such data as device parameters and device interconnects. The central control computers thus receive updated data as soon as the component is connected and do not have to wait until the database is updated by central control personnel.

The joint Electric Power Research Institute (EPRI) and U.S. Department of Defense (DOD) program, through the Complex Interactive Networks/Systems Initiative (CIN/SI), showed that the grid can be operated close to the limit of stability given adequate situational awareness combined with more secure communication and better controls. A smart grid would be able to meet these awareness, communication, and control needs.

As part of enabling a self-healing grid, we have developed adaptive protection and coordination methods that minimize the impact on overall system performance (in terms of both load dropped and robust rapid restoration).

Note that, although computation is now heavily used in all levels of the power network (e.g., for planning and optimization, fast local control of equipment, and processing of field data), coordination across the network happens on a slower time scale, based on a system of operation developed in the 1960s. Some coordination occurs under computer control, but much of it is still based on telephone calls between system operators at the utility control centers, especially during emergencies.

In any situation subject to rapid changes, completely centralized control requires multiple, high-data-rate, two-way communication links; a powerful central computing facility; and an elaborate operations control center. However, all of these components are susceptible to disruption at the very time when they are most needed (i.e., when the system is stressed by natural disasters, purposeful attack, or unusually high demand).

When failures occur at various locations in such a network, the whole system breaks into isolated "islands," each of which must then fend for itself. However, in the proposed smart grid, in which the intelligence is distributed throughout the components in the system acting as independent agents, components in each island have the ability to reorganize themselves and make efficient use of available local resources until they are able to rejoin the network. A network of local controllers can act as a parallel, distributed computer, communicating via microwaves, optical cables, or the power lines themselves and intelligently limiting their messages to only that information necessary to achieve global optimization and facilitate recovery after failure.

The EPRI/DOD CIN/SI, which concluded in 2001, aimed to develop modeling, simulation, analysis, and synthesis tools for robust, adaptive, and reconfigurable control of the electric power grid and infrastructures connected to it. In part, this work showed that adequate situational awareness combined with better sensing of system conditions, communication, and controls would allow the grid to be operated efficiently close to the limit of stability. Grid operators often make quick decisions under considerable stress. Given that, in recent decades, we have reduced the generation and transmission capacity, we are indeed flying closer to the edge of the stability envelope.

As an example, EPRI's IntelliGrid program seeks to develop practical solutions based on the foundation established by the CIN/SI. One aspect of this program is aimed at enabling grid operators greater look-ahead capability and foresight, overcoming limitations of the current schemes, which, at best, have a $>30$-s delay in assessing system behavior-analogous to driving a car by looking into the 
rear-view mirror instead of at the road ahead. This tool using advanced sensing, communication, and a software module was proposed during 2000-2001, and the program was initiated in 2002 under the Fast Simulation and Modeling (or FSM) program. This advanced simulation and modeling program promotes greater grid self-awareness and resilience in times of crisis in three ways: by providing faster-than-realtime look-ahead simulations (analogous to master chess players rapidly expanding and evaluating their various options under time constraints) and thus avoiding previously unforeseen disturbances; by performing what-if analyses for largeregion power systems from both operations and planning points of view; and by integrating market, policy, and risk analyses into system models and quantifying their integrated effects on system security and reliability.

Such an approach provides an expanded stability region with larger operational range; as the operating point nears the limit to how much the grid can be adapted (e.g., by automatically rerouting power and/or dropping a small amount of load or generation), rather than cascading failures and large-scale regional system blackouts, the system will be reconfigured to minimize the severity/size of outages, to shorten the duration of brownouts/blackouts, and to enable rapid/efficient restoration.

The simplest kind of distributed control would combine remote sensors and actuators to form regulators (e.g., intelligent electronically controlled secure devices) and adjust their set points or biases with signals from a central location. Such an approach requires a different way of modeling - of thinking about, organizing, and designing - the control of a complex, distributed system. Recent research results from a variety of fields, including nonlinear dynamical systems, artificial intelligence, game theory, and software engineering, have led to a general theory of complex adaptive systems (CASs). Mathematical and computational techniques originally developed and enhanced for the scientific study of CASs provide new tools for the engineering design of distributed control so that both centralized decision-making and the communication burden it creates can be minimized. The basic approach to analyzing a CAS is to model its components as independent adaptive software and hardware "agents," partly cooperating and partly competing with each other in their local operations while pursuing global goals set by a minimal supervisory function.

$\mathrm{CIN} / \mathrm{SI}$ has developed, among other things, a new vision for the integrated sensing, communications, and control of the power grid. Some of the pertinent issues are why/how to develop controllers for centralized as opposed to decentralized control and how to address adaptive operation and robustness to disturbances that include various types of failures. As expressed in the July 2001 issue of Wired magazine: "The best minds in electricity R\&D have a plan: Every node in the power network of the future will be awake, responsive, adaptive, price-smart, eco-sensitive, real-time, flexible, humming - and interconnected with everything else."

\section{Enabling Technologies}

During the past 10 years, we have investigated whether there are leading applications of science and technology outside the traditional electric energy industry that might apply in meeting and shaping consumer needs. These applications could include entirely new technologies, not part of the portfolio of traditional electricity solutions and not identified in other tasks, which could be potentially available as well. Some technology areas include materials and devices, such as nanotechnology, microfabrication, advanced materials, and smart devices; meso- and microscale devices and sensors and networks; advances in information science, such as algorithms, artificial intelligence, systems dynamics, network theory, and complexity theory; bioinformatics, biomimetics, biomechatronics, and systems biology; enviromatics, such as the development of new methodologies and the use of state-of-the-art information technology for improved environmental applications; other industries - moving to a wireless world - such as transportation, telecommunications, digital technologies, sensing, and control; markets, economics, policy, and environment; and end-to-end infrastructure, from fuel supply to end use.

We believe that six technology platforms, namely, sensors, biotechnology, smart materials, nanotechnology, fullerenes, and information technology, could prove particularly important in solving the challenges facing the electric energy industry. These platforms were selected on the basis of past technology roadmapping efforts to identify key underlying technologies, and the emphasis is primarily on long-term, limit-breaking developments. Higher-temperature alloys for turbines and steam generator components, for example, are certainly important, but their development is likely to follow from conventional, near-term refinement work, so they are not discussed here; on the other hand, more-innovative solutions to heat-based turbine problems - and much larger improvements - might result from longer term research on biomimetic ceramics or fullerene composite materials. The outlook and future possibilities for some of these technology platforms are summarized next.

\section{Sensors}

Industry has always been dependent on measurement instruments to ensure safe, efficient processes and operations, and today, almost every engineering system incorporates sophisticated sensor technology to achieve these goals. However, an increased focus on cost and efficiency, along with the growing complexity of industrial processes and systems, have placed new demands on measurement and monitoring technology: operators are requesting more accurate data on more variables from more system locations in real time. The power industry, with its large capital investment in expensive machinery and its complicated, extremely dynamic delivery system, has an especially pressing need for advanced sensors that are small enough to be used in distributed applications throughout power systems. Continued development of digital control systems to replace far-less-accurate analog and pneumatic controls is a key research focus. Sensors that can accurately detect and measure a wide range of chemical species, such as $\mathrm{CO}_{2}, \mathrm{NO}_{x}$, and $\mathrm{SO}_{2}$, are needed, as are sensors and gauges robust enough to withstand the harsh temperatures and chemical environments characteristic of power plants. Advanced fiber-optic sensors - devices based on sapphire fibers or fiber Bragg gratings, for instance - are especially important because of their versatility, small size, and freedom from magnetic interference. Another possibility involves using wireless sensor networks, as discussed in the accompanying sidebar by Steingart et al. Overcoming today's limitations on temperature, robustness, versatility, and size will facilitate fulfillment of a number of long-standing power system needs, including realtime characterization of plant emissions and waste streams, distributed measurement of transformer winding temperatures, and on-line monitoring of $\mathrm{pH}$ in steam plant circulation water.

\section{Smart Materials}

Smart materials are necessary in the future power grid to give it the ability to self-recover with fast response in milliseconds under outage events or terrorist attacks. To accomplish this level of self-recovery, it is necessary to make each component intelligent. Such local, autonomous control will make the system much more resilient to multiple contingencies. Control components must be reconfigurable power electronic devices 
with a distributed controller to function as high-speed switches to redirect power flow. They must also have an overall architecture suitable to the new control/communication paradigm. Finally, these components must possess a fault-tolerant, agentbased collaborative intelligence at the lower level of the system to tremendously expedite the response to contingency events.

One class of materials known as smart materials and structures (SMSs) has the unique capability to sense and physically respond to changes in the environment - changes in temperature, $\mathrm{pH}$, or magnetic field, for example. Generally consisting of a sensor, an actuator, and a processor, SMS devices based on such materials as piezoelectric polymers, shape-memory alloys, hydrogels, and fiber optics can function autonomously in an almost biological manner. Smart materials have already appeared in a number of consumer products and are being studied extensively for aircraft, aerospace, automotive, electronics, and medical applications. In the electric power field, SMSs hold promise for real-time condition assessment of critical power plant components, allowing continuous monitoring of remaining life and timely maintenance and component replacement. Control of power plant cycle chemistry could be done rapidly and automatically with smart systems to inject chemicals that counter pollutants or chemical imbalances. Control of $\mathrm{NO}_{x}$ creation in boilers could be accomplished by adjusting the combustion process with sensor and activation devices distributed at different boiler locations.

For the wires used in the electric power industry, smart materials could be utilized to monitor the condition of conductors, breakers, and transformers to avoid outages. Smart materials could also be used to avoid potentially catastrophic subsynchronous resonance in generating units and to adjust transmission line loads according to real-time thermal measurements. Critical capability gaps are related to integrating smart materials into sensors, actuators, and processors; embedding the SMS components into the structure to be controlled; and facilitating communication between smart structure components and the external world.

The growing list of smart materials encompasses a number of different physical forms that respond to a wide variety of stimuli. Examples include the following:

- Piezoelectric ceramics and polymers are materials, such as lead zirconate titanate ceramics and polyvinylidene fluoride polymers, that react to physical pressure. They can be used as either sensors or actuators, depending on their polarity.

- Shape-memory alloys are metal alloys, such as nitinol, that can serve as actuators by undergoing a phase transition at a specific temperature and reverting to their original, undeformed shape.

- Shape-memory polymers are a class of elastomer-like polymers, such as polyurethane, that actuate by relaxing to their undeformed shape when heated above their glass transition temperatures.

- Conductive polymers are polymers that undergo dimensional changes upon exposure to an electric field. These versatile materials can be used not only as sensors and actuators, but also as conductors, insulators, and shields against electromagnetic interference.

- Electrorheological fluids are materials containing polarized particles in a nonconducting fluid that stiffens when exposed to an electric field. As such, they can be used in advanced actuators.

- Magnetorestrictive materials include molecular ferromagnetic materials and other metallic alloys that change dimensions when exposed to a magnetic field.

- Polymeric biomaterials are synthetic, muscle-like fibers, such as polypeptides, that contract and expand in response to temperature or chemical changes in their environment.
- Hydrogels are cross-linked polymer networks that change shape in response to changes in electric fields, light, electromagnetic radiation, temperature, or $\mathrm{pH}$.

- Fiber optics are fine glass fibers that signal environmental change through analysis of light transmitted through them. Perhaps the most versatile sensor material, optical fibers can indicate changes in force, pressure, density, temperature, radiation, magnetic field, and electric current.

These materials, when matched to an appropriate application, provide the base functionality for both simple and higherlevel smart structures and systems. Sensory structures, such as optical fibers embedded in concrete bridge support pillars, only furnish information about system states; with no actuator, they are able to monitor the health of the structure but cannot physically respond to improve the situation. Adaptive structures contain actuators that enable controlled alteration of system states or characteristics; electrorheological materials, for example, can damp out vibrations in rotating mechanical systems when an electric field is applied. Controlled structures provide feedback between sensors and actuators, allowing the structure to be fine-tuned continuously and in real time; for example, aircraft wings outfitted with piezoelectric sensors and actuators can be programmed to subtly change shape to avoid flutter under problematic wind conditions. A grid operator is similar to a pilot flying an aircraft, monitoring how the system is being affected and having a solid sense of how to steer it in a stable fashion.

Examples of higher-level smart structures and systems that can be built from smart materials and utilized in the grid include flexible alternating-current transmission, high-voltage directcurrent transmission systems, and dynamic line rating.

Flexible ac transmission (FACTS) devices are a family of solid-state power control devices that provide enhanced dynamic control of the voltage, impedance, and phase angle of high-voltage ac transmission lines. FACTS controllers act like integrated circuits - but scaled up by a factor of 500 million in power. By applying FACTS devices, utilities can increase the capacity of individual transmission lines by up to $50 \%$ and improve system stability by responding quickly to power disturbances.

There is a need to reduce the costs of FACTS technology to provide for broader use. One method for reducing the costs is to replace the silicon-based power electronics with wide-bandgap semiconductors such as silicon carbide ( $\mathrm{SiC}$ ), gallium nitride $(\mathrm{GaN})$, and diamond.

High-voltage direct-current (HVDC) transmission systems are based on the rectification of the generated ac and then conversion back to ac at the other end of the transmission line. Modern systems are based on thyristor valves (solid-state power control devices) to perform the ac/dc/ac conversions. Conventional HVDC transmission systems have been built with power transfer capacities of $3000 \mathrm{MW}$ and $\pm 600 \mathrm{kV}$. A new class of HVDC converter technology, referred to as voltage source converters, has been introduced in the past few years. These devices are based on gate turn-off switching technology or insulated gate bipolar transistors and are capable of higher switching frequencies. HVDC transmission is used for long-distance bulk power transmission over land or for long submarine cable crossings. Altogether, there are more than 35 HVDC systems operating or under construction in the world today. The longest HVDC submarine cable system in operation today is the $250-\mathrm{km}$ Baltic Cable between Sweden and Germany.

Dynamic line rating could enable increased power flow over existing transmission lines. Specifically, the maximum power that can be carried by a transmission line is ultimately determined by how much the line heats up and expands. The "thermal 
rating" of a line specifies the maximum amount of power it can safely carry under specific conditions without drooping too much. Most thermal ratings today are static in the sense that they are not changed through the year. For such ratings to be reliable, they must be based on worst-case weather conditions, including both temperature and wind velocity. In contrast, dynamic line ratings use real-time knowledge about weather or line sag to determine how much power can be transmitted safely. Typically, a dynamically monitored line can increase its allowable power flow (ampacity) by $10-15 \%$ over that allowed by static ratings.

In the future, smart materials and structures are expected to appear in applications that span the entire electric power system, from power plant to end user. Smart materials, in their versatility, could be used to monitor the integrity of overhead conductor splices, suppress noise from transformers and large power plant cooling fans, reduce cavitation erosion in pumps and hydroturbines, or allow nuclear plants to better handle structural loads during earthquakes.

\section{Advanced Hardware}

Incorporating smart materials and higher-level smart structures and systems into the grid will require the development of advanced hardware components. These include advanced meters, advanced sensors and monitors, advanced motors (including superconducting motors), advanced transformers (including the concept of a universal transformer that would be a standardized portable design, a FACTS phaseshifting transformer capable of controlling power flow, and next-generation transformers using solid-state devices and high-temperature superconductors), power electronics (including FACTS, solid-state breakers, switchgear, and fault current limiters), computers and networks, mobile devices, and smart equipment and appliances.

Advanced hardware includes both cable and storage options. Among advanced cables are gas-insulated lines for underground cables (for costs, see Reference 33), advanced composite conductors (which are lighter and carrying more current than the current ACSR conductors), and high-temperature superconductors. It is pointed out that these could also revolutionize generators, transformers, and fault current limiters. Potential developments in electric storage include superconducting magnetic energy storage (SMES), advanced flywheels (see the sidebar in the article by Whittingham in this issue) using composites and/or superconductors for higher efficiency and capacity, flow batteries that charge and discharge fluid between tanks, and liquid molten sulfur batteries built to utility scale.

\section{Nanotechnology}

The theme of the development of nanotechnology in energy application technology is geared toward two main directions: nanomaterials for energy storage and nanotechnology for energy saving. Owing to the advantages of high reactivity, large surface area (200-2000 $\left.\mathrm{m}^{2} / \mathrm{g}\right)$, self-assembly ( 1-3-nm active catalyst), super crystal characteristics $(\sim 10-30-\mathrm{nm}$ nanostructures), and special opto-electronic effects of nanomaterials for energy saving, several countries are heavily engaged in the development of energy-related nanomaterials.

There is an expectation that nanotechnologies will enable the development of power storage systems with energy densities that are at least several times higher than those of current batteries. Because of the small dimensions (5-20 nm), high specific surface areas, and special optical properties of nanomaterials, nanotechnology for energy saving is expected to increase with the contact area of the medium. This will shorten response time and improve thermal conductivity by a factor of two. Nanotechnology applications for energy storage include using nanoparticles and nano- tubes for batteries and fuel cells. Nanotechnology is being used to better the performance of rechargeable batteries through the study of molecular electrochemical behavior. Newly patented lithium ion batteries that use nanosized lithium titanate can provide 10-100 times greater charging/discharging rates than current conventional batteries. Other new batteries that apply nanotechnology could provide added power and storage capabilities by applying a concept based on mechanical resonance using a single microelectromechanical systems (MEMS) device; such devices use the combined technology of computers and mechanical devices to improve the power density, offering significant benefits for portable equipment.

\section{Fullerenes}

Discovered in the 1980s, fullerenes are a type of carbon molecule that exhibit extraordinary properties, including high strength, toughness, and both metallic and semiconducting electrical characteristics. The soccer-ball-patterned $\mathrm{C}_{60}$ molecule and the cylindrical carbon nanotube are considered by many to be the ultimate materials, and although only small amounts of fullerenes have yet been produced, researchers have suggested many potential applications. Most of these involve the carbon nanotube, a long, hollow string with tremendous tensile strength that could be wound into the strongest structural cable ever made. Use of shorter nanotube strings in metal, ceramic, or polymer composites would create stronger, lighter, more versatile materials than are currently available in any form. Electrical applications range from highly conductive (and perhaps superconductive) wires and cables to electron emitters in flat-panel displays to magnetic recording media for data storage. Because nanotubes are incredibly thin and have such versatile electrical properties, they are seen as ideal building blocks for nanoscale electronic devices. Realization of such possibilities is highly dependent on developing processes for producing high-quality fullerenes in industrial quantities at reasonable cost and in finding ways to manipulate and orient nanotubes into regular arrays. Cost will almost certainly determine whether fullerenes will become a true universally used material or an esoteric, highcost/high-value option for specialized applications.

\section{Future Opportunities and Challenges}

To highlight further opportunities where science and technology from other industries could possibly be identified to fill these gaps, the following issues must be addressed:

- low-cost, practical electric and thermal energy storage;

- microgrids, ac and dc, including both self-contained, cellular, and universal energy systems and larger building- or campus-sized systems;

- advanced (post-silicon) power electronics devices (valves) to be embedded into flexible ac and dc transmission and distribution circuit breakers, short-circuit current limiters, and power electronics-based transformers;

- power electronic-based distribution network devices with integrated sensors and communications;

- fail-safe communications that are transparent and integrated into the power system;

- cost-competitive fuel cell;

- low-cost sensors to monitor system components and to provide the basis for state estimation in real time;

a cost-effective integrated thermal storage (heating and cooling) devices;

- thermal appliances that provide "plug-and-play" capability with distributed generation devices;

- high-efficiency lighting, refrigerators, motors, and cooling;

- enhanced portability through improved storage and power conversion devices; 
- efficient, reliable, cost-effective plug-in hybrid electric vehicles (PHEVs);

- technologies and systems that enable "hardened" end-use devices;

- conductors that enable greatly increased power flow capability;

- smart, green, zero-energy buildings; and

- thermoelectric devices that convert heat directly to electricity.

However, these technologies will require sustained funding and commitment to research, development, and demonstration. Given the state of the art in electricity infrastructure security and control, creating a smart grid with self-healing capabilities is no longer a distant dream; considerable progress has been made toward this goal. The cost of a self-healing smart grid will not be cheap-some estimates are as much as $\$ 10-13$ billion per year needed for a period of 10 years or more for real-world testing and installation. Yet, the price of electrical failure, estimated at over $\$ 80$ billion per year, is not cheap either.

There are signs too that the U.S. Congress and government recognize the need for action. Recently, the White House Office of Science and Technology Policy (OSTP) and the U.S. Department of Homeland Security (DHS) declared the selfhealing infrastructure to be one of three strategic thrust areas for the National Plan for R\&D in Support of Critical Infrastructure Protection. ${ }^{28}$

However, considerable technical challenges and several economic and policy issues remain to be addressed. At the core of the power infrastructure investment problem lie two paradoxes of restructuring, one technical and one economic. Technically, the fact that electricity supply and demand must be in instantaneous balance at all times must be resolved with the fact that new power infrastructure is extraordinarily complex, time-consuming, and expensive to construct. Economically, the theory of deregulation aims to achieve the lowest price through increased competition. However, the market reality of electricity deregulation has often resulted in a business-focused drive for maximum efficiency to achieve the highest profit from existing assets rather than in lower prices or improved reliability. Both the technical and economic paradoxes can be resolved through knowledge and technology.

Given economic, societal, and quality-of-life issues and the ever-increasing interdependencies among infrastructures, a key challenge before us is whether the electricity infrastructure will evolve to become the primary support for the 21 st century's digital society - a smart grid with self-healing capabilities - or be left behind as a 20th century industrial relic.

\section{Acknowledgments}

Most of the material and findings presented here were developed while the authors were at the Electric Power Research Institute (EPRI) in Palo Alto, California. We gratefully acknowledge the support and feedback from numerous colleagues at EPRI, universities, industry, and government agencies. In addition, Massoud Amin gratefully acknowledges support of his work by EPRI, NSF's Cyber Trust, and the ORNL Sensor Net Projects.

\section{References}

1. M. Amin, IEEE Secur. Priv. Mag. 1 (5), 19 (September/October 2003).

2. M. Amin, IEEE Comput. Mag. Secur. Priv. (April 2002).
3. M. Amin, IEEE Comput. Appl. Power 14 (1), 20 (January 2001)

4. M. Amin, IEEE Comput. Mag. 33 (8), 44 (August 2000).

5. M. Amin, IEEE Control Syst. Mag. Control Complex Netw. 21 (6) (December 2001); and 22 (1) (February 2002).

6. DOE, National Transmission Grid Study (U.S. Department of Energy, May 2002).

7. Energy Information Administration, DOE, Annual Energy Outlook 2003; www.eia.doe.gov/oiaf/aeo/figure 3.html (accessed January 2008).

8. EPRI 2003, Electricity Technology Roadmap: Synthesis Module on Power Delivery System and Electricity Markets of the Future (EPRI, Palo Alto, CA, July 2003).

9. EPRI 1999, Electricity Technology Roadmap: 1999 Summary and Synthesis, Technical Report Cl-112677-V1, 160 (EPRI, Palo Alto, CA, 1999).

10. Committee hearing of the House Committee on Energy and Commerce, Blackout 2003: How Did It Happen and Why? (September 3-4, 2003); http:// energycommerce.house.gov (accessed January 2008).

11. Critical Foundations: Protecting America's Infrastructures, The report of the President's Commission on Critical Infrastructure Protection (Washington

DC, October 1997).

12. EPRI 2003, Complex Interactive Networks/Systems Initiative: Final Summary Report-Overview and Summary Final Report for Joint EPRI and U.S. DOD University Research Initiative, 155 (EPRI, Palo Alto, CA, December 2003).

13. EPRI 2001, Electricity Infrastructure Security Assessment I-II (EPRI, Palo Alto, CA, November and December 2001).

14. EPRI 2000, Communication Security Assessment for the United States Electric Utility Infrastructure 1001174, 4 (EPRI Report, December 2000)

15. In a report from the Edison Electric Institute, "Meeting U.S. Transmission Needs" (July 2005).

16. F.F. Hauer, J.E. Dagle, Review of Recent Reliability Issues and System Events, Consortium for Electric Reliability Technology Solutions (Transmission Reliability Program, Office of Power Technologies, U.S. DOE, August 30, 1999).

17. Kundur, Power System Stability and Control. EPRI Power System Engineering Series (McGraw-Hill, Inc., 1994).

18. T.E. Dy Liacco, IEEE on PAS 517 (May 1967).

19. L.H. Fink, K. Carlsen. IEEE Spectr. 48 (March 1977).

20. M. Amin, Special Issue of the Proc. IEEE Energy Infrastructure Def. Syst 93 (5) (May 2005).

21. M. Samotyj, C. Gellings, M. Amin, GIGRE/IEEE-PES Symp. on Quality and Security of Electric Power Delivery 10 (Montreal, October 7-10, 2003)

22. M. Amin, B.F. Wollenberg, IEEE Power Energy Mag. 3 (5), 34 (September/ October 2005).

23. M. Amin, Special Issue of Proc. IEEE 93 (5), 857 (May 2005).

24. M. Amin, C. Gellings, Energy 31 (6-7), 967 (May-June 2006).

25. M. Amin, L.W. Carlson, C. Gellings, Galvin Electricity Initiative: Technology Scanning, Mapping and Foresight, 70 (EPRI, Palo Alto, CA and Galvin Electricity Project, Inc., Chicago, IL, March 2006).

26. Brendan Kirby of ORNL's Engineering Science and Technology Division contributed a paper entitled "Reliability Management and Oversight" and coauthored another, "Transmission Planning and the Need for New Capacity" to a DOE National Transmission Grid Study, which consisted of six issue papers published and released in March 2002.

27. G. Newaz, D. Bigg, and R. Eiber of Battelle's Columbus Division presented a report to EPRI entitled "Structural Composite Cores for Overhead Transmission Conductors" in April, 1987 (EPRI Report EM-5110, Research Project 2426-9).

28. M. Amin, P.F. Schewe, Sci. Am. 60 (May 2007)

29. P.F. Schewe, The Grid: A Journey through the Heart of Our Electrified World (Joseph Henry Press, 2007).

30. E.J. Lerner, The Industrial Physicist, American Institute of Physics 9, 8 (October/November 2003).

31. According to a U.S. Department of Energy Office of Electricity Delivery and Energy Reliability GridWorks Overview of the Electric Grid dated August 2005.

32. North American Electric Reliability Council (NERC) Disturbance Analysis Working Group (DAWG) database.

33. Edison Electric Institute, "'Out of Sight, Out of Mind?' A Study on the Costs and Benefits of Undergrounding Overhead Power Lines" (January 2004).

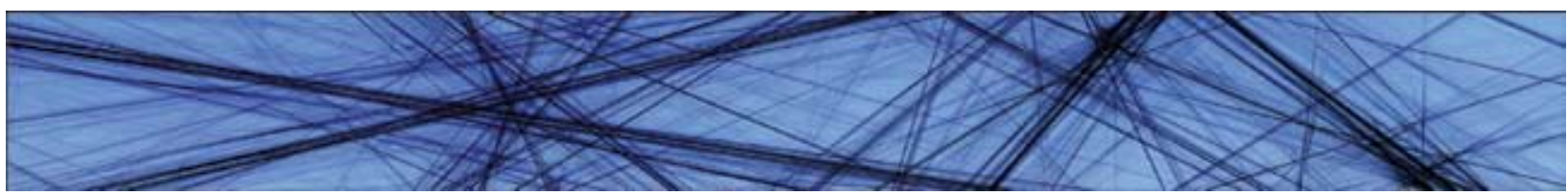




\section{Cut Big Problems Down to Size}

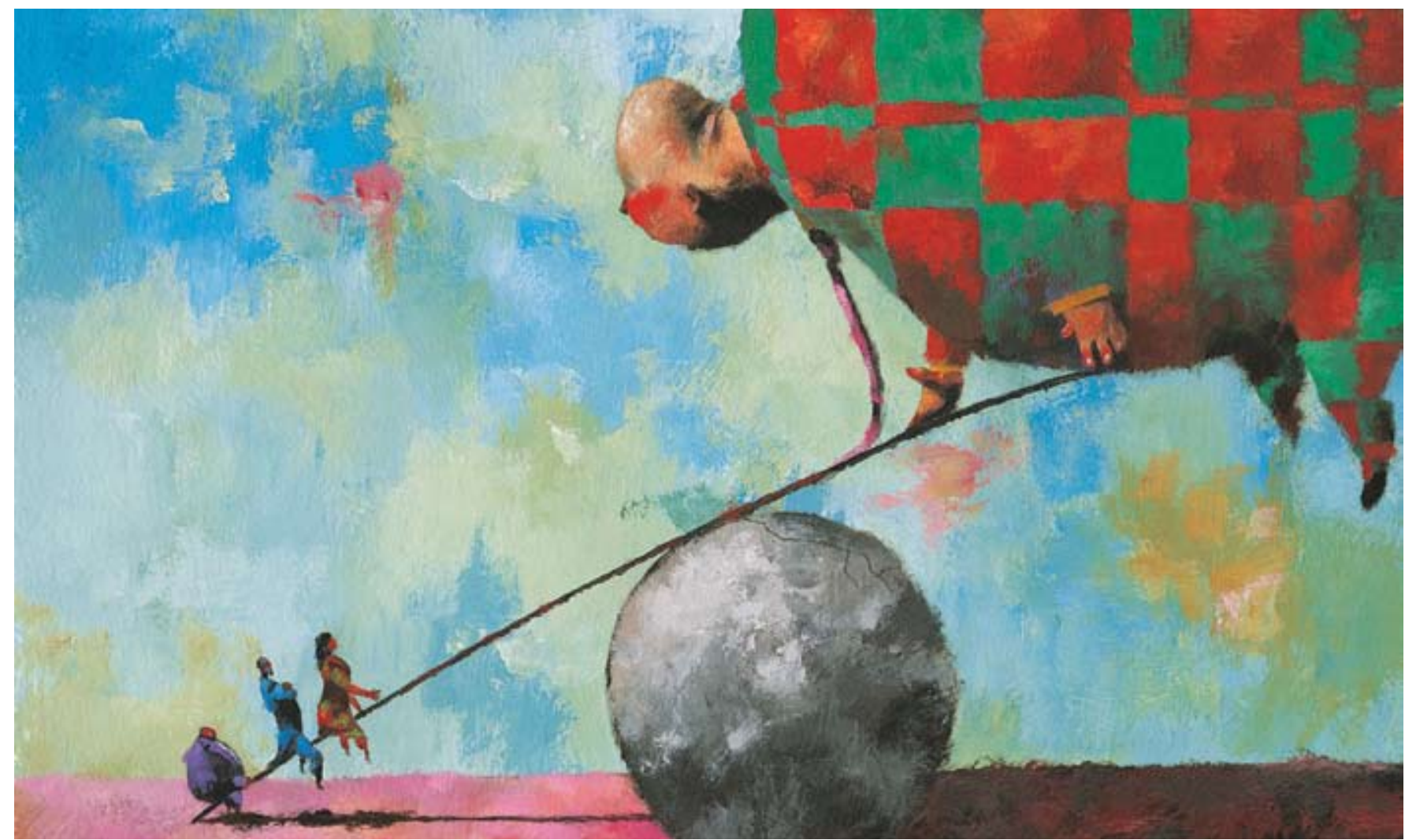

\section{With Innovative AFM Solutions}

rinsic bowing in atomic force microscopy messing up your flat sample images? Having difficultly measuring overhang structures? Can't get the spatial and thermal resolution you need to measure heat dispersion? Major problems, indeed.

\section{No Crosstalk. No Problems.}

While some AFMs still use 1980's technology, we've leveraged innovations in hardware, software and probes to deliver scans with astounding clarity and accuracy. And our XE Family ${ }^{\text {TM }}$ of AFMs and new NSOM Systems repeat those results sample after sample.

\section{A Decade of "Can Do."}

Park Systems may be a new name. But we're not a new company. In fact, we've been around since the AFM was invented, winning one industry award after another. Call us at 408-986-1110 and we'll show you Excellence in Nanometrology.

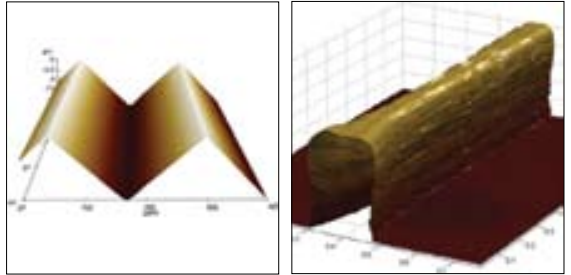

Angle Measurements Photoresist Overhang

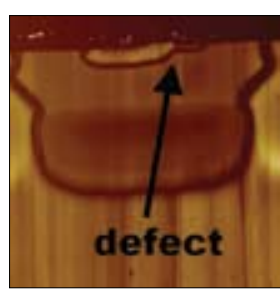

Dopant Imaging

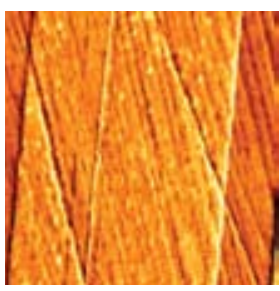

Disk Head Imaging ( $2 \mu \mathrm{m} \times 2 \mu \mathrm{m})$ Topographical Image Thermal Image

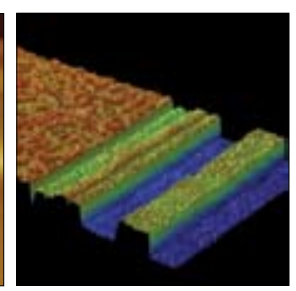

Hard Disk Slider

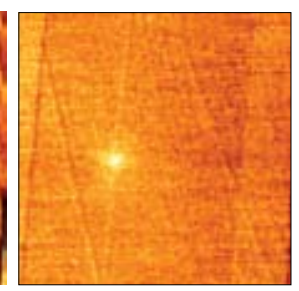$$
\text { The }
$$
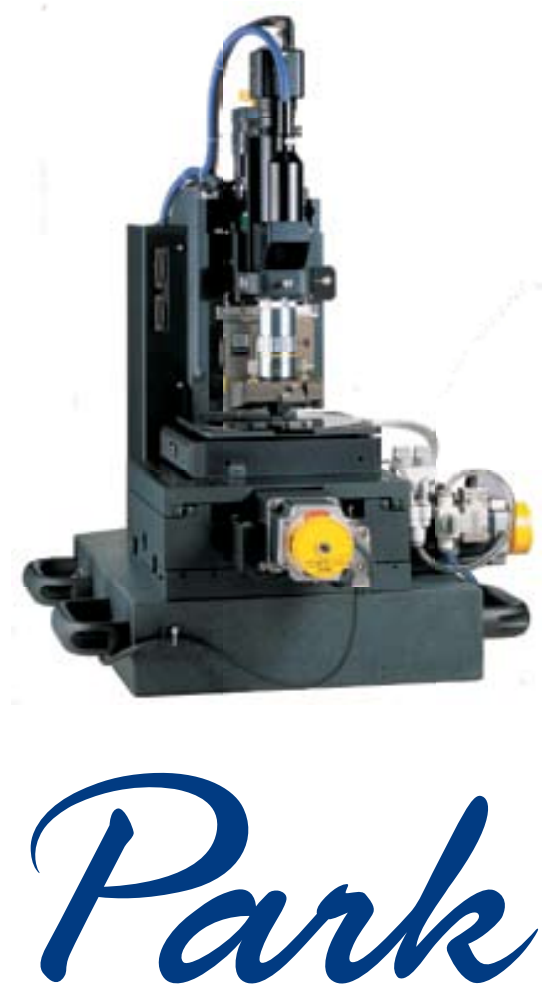

\section{S Y S T E M S}

www.parkAFM.com email us at info@parkAFM.com 